\title{
Conditional sampling, bursting, and the intermittent structure of sensible heat flux
}

\author{
Gabriel G. Katul, ${ }^{1}$ John Albertson, ${ }^{2}$ Marc Parlange, ${ }^{2}$ Chia-Ren Chu, ${ }^{3}$ and Han \\ Stricker ${ }^{4}$
}

\begin{abstract}
Sensible heat flux measurements were carried out to examine the characteristics related to frequency and duration of extreme flux events above a desert surface. First, the flux bursts were identified by a modified hyperbolic hole "quadrant analysis" in which the threshold criterion depends on the mean and standard deviation of the flux time statistics. Second, the frequency distribution of the extreme flux burst length was examined. It was found that the frequency distribution of the burst length is well approximated by two- parameter power laws which account for two distinct types of bursting phenomena: one is organized, coherent, and has a relatively long duration, while the other is short lived, less organized, and has a relatively short duration. Linear regression analysis was used to determine the two power exponents of the burst length probability density function. Both exponents compare favorably with other reported measurements on concentration bursting in dispersing plumes from an elevated source. The cutoff burst length from one power law to the next also matches other studies. A strong correlation between bursting frequency and flux integral time scales was observed under a variety of atmospheric stability and surface wetness conditions. This strong correlation suggested a relationship between atmospheric stability and the mean time interval between bursts. It was found that the variation in the interval between bursts for the dynamic and dynamic convective sublayers is strongly related to the variation in stability. This was not the case for near-convective and stable atmospheric conditions.
\end{abstract}

\section{Introduction}

A major part of the energy that drives the growth and decay of the planetary boundary layer above arid regions and deserts comes from sensible heat flux. Currently, deserts and desert-like regions occupy about $20 \%$ of the total land area, and thus interest in characterizing the sensible heat flux from desert-like surfaces has received great attention in climate and atmospheric models [Dickinson, 1983; Sellers, 1992]. A big portion of sensible heat flux is generated close to the ground surface and transported into the atmosphere by the turbulent air flow.

Although the air flow above the desert surface is in a continous state of turbulence, the flux of heat appears to be very intermittent. This ubiquitous intermittency feature of heat fluxes results in peaks much larger than the mean turbulent flux. Interest in identifying specific events responsible for this intermittency, their frequency as well as

${ }^{1}$ School of the Environment, Duke University, North Carolina. ${ }^{2}$ Hydrologic Science, University of California, Davis.

${ }^{3}$ Department of Civil Engineering, National Central University, Chung-Li, Taiwan.

${ }^{4}$ Department of Water Resources, Agricultural Engineering, Wageningen, Netherlands.

Copyright 1994 by the American Geophysical Union.

Paper number 94JD01679.

0148-0227/94/94JD-01679\$05.00 their duration, has therefore received great attention in meteorology, hydrology, and other engineering disciplines [e.g., Duncan and Shuepp, 1992; Paw U et al., 1992; Gao et al., 1992; Mahrt, 1991; Shaw, 1985, Chen and Blackwelder, 1978; Blackwelder and Kaplan, 1976; Antonia et al., 1979). It is well recognized that these extreme events are relatively organized and contribute more than $70 \%$ to the mean turbulent sensible heat flux. Thus a better understanding of the occurrence, duration, and frequency of these events is necessary.

Many techniques have been developed to identify these extreme flux events and their contribution to the mean turbulent flux. One popular technique, known as conditional sampling, has been very effective in delineating extreme flux events in time measurements of turbulent fluxes. Conditional sampling methodologies, based on the so-called "quadrant analysis", were successful in identifying complex structures responsible for organized features such as plumes and thermals in buoyancy-driven turbulence or organized eddy formation in shear-driven turbulence [e.g. Antonia, 1981; Shaw et al., 1983; Shaw, 1985; Raupach, 1981; Raupach et al., 1991; Grossman, 1984]. Other studies successfully established formal relations between the "quadrant analysis" and higher order closure models making this conditional sampling scheme even more attractive in analyzing turbulence measurements (for example, see the pioneering work of Nakagawa and Nezu [1977, 1981] on bursting in open channel flows).

The term "quadrant analysis" refers to a set of analyzing techniques that are applied to scatter plots formed by two turbulent quantities such as vertical velocity fluctuations $\left(w^{\prime}\right)$ and temperature fluctuations $\left(T^{\prime}\right)$. Here we assume that the 
temperature and vertical velocity time series measurements can be decomposed, without ambiguity, into a mean and a turbulent fluctuating part. The four quadrants defined by the Cartesian axes of the scatter plot commonly represent four modes of turbulent transport: (e.g., $w^{\prime}>0$ and $T^{\prime}>0, w^{\prime}<0$ and $T^{\prime}>0, w^{\prime}>0$ and $T^{\prime}<0$, and $w^{\prime}<0$ and $\left.T^{\prime}<0\right)$. One method of identifying extreme flux events in quadrant analysis is known as the "hyperbolic hole." This method identifies extreme flux events as events outside the hyperbola $w^{\prime} \times T^{\prime}=C\left|\left\langle w^{\prime} T^{\prime}\right\rangle\right|$ in all four quadrants, where $C$ is some arbitrary constant, and $\langle>$ is the ensemble averaging operator. Momentum and heat flux measurements by Shaw [1985] above and within tree canopies suggest that a value of $C=4$ is well suited for identifying extreme flux events. Carbon dioxide and water vapor flux measurements by Duncan and Shuepp [1992] reinforce Shaw's [1985] conclusion regarding the threshold for $C$.

In this study, we develop and use a modification of the "quadrant analysis" to identify extreme sensible heat flux events. The distinction between the proposed method and other hyperbolic hole methods is the dependence of $C$ on the standard deviation of the instantaneous heat flux time series. Once the extreme events are identified in all time series measurements, an ensemble of extreme events (or bursts) can be formed.

Using the constructed ensemble, our objective here is to investigate a statistical property of key importance in landsurface fluxes: the frequency/duration of occurrence of the extreme sensible heat flux events. The duration and frequency of occurrence of these events are crucial in describing the local structure of sensible heat. Variables parameterizing the local structure of sensible heat are important since they permit (1) the construction of more realistic closure models for the time-averaged Navier-Stokes equations, (2) the comparisons between measurements and numerically simulated turbulence (as in large eddy simulations), and (3) the determination of the minimum sampling period for measuring the heat flux in many field experiments.

To examine these statistical properties, $T^{\prime}$ and $w^{\prime}$ were measured in the atmospheric surface layer (ASL) over a dry smooth lakebed and over a uniform bare soil surface. These measurements were conducted for a variety of atmospheric stability and surface wetness conditions. Details regarding the experimental setup and measurements are outlined below.

\section{Experiments}

The experiments were carried out at two sites. The first site is above a dry lakebed in Owens Valley, and the second site is a research plot at the University of California, Davis Campbell Tract facility.

\subsection{Owens Lake}

The temperature $(T)$ and vertical velocity $(w)$ measurements were carried out from June 20 to July 2, 1993 over a dry lakebed (Owens lake) in Owens Valley, California. The lakebed is part of a larger basin bounded from the west by the White and Inyo Mountains and from the east by the Sierra Nevada. The sensible heat flux measurements were performed on the northeast portion of the lakebed (elevation of $1100 \mathrm{~m}$ ). The lakebed surface surrounding the site is a uniform heaved sandy soil extending several kilometers in all directions. The momentum roughness length $\left(z_{0}\right)$ of the lakebed is $0.2 \mathrm{~mm}$ (Katul [1994]).

The vertical velocity component was measured using a triaxial ultrasonic anemometer (Gill Instruments, model 1012R2) to an accuracy of $\pm 1 \%$ (Katul et al., 1994). The sonic anemometer path length $d_{s l}$ is $0.149 \mathrm{~m}$, and the maximum sampling frequency is $56 \mathrm{~Hz}$. The maximum sampling frequency was used at this site. To insure that the steady state assumption is valid, a sampling period of $15 \mathrm{~min}$ was chosen. This yielded 50,400 measurements of $w$ and $T$. The sonic anemometer height was varied between $1.5 \mathrm{~m}$ and $3.5 \mathrm{~m}$.

The absolute temperature $(T)$ was computed directly from the measured fluctuations in the speed of sound $\left(c_{s}\right)$ using

$$
T=\frac{c_{s}^{2}}{\alpha R_{d}}
$$

where $\alpha=C_{p} / C_{v}$, where $C_{p}$ and $C_{v}$ are the specific heat capacities of dry air under constant pressure and volume, respectively, and $R_{d}$ is the gas constant for dry air [see Suomi and Businger, 1959; Wyngaard, 1981; Friehe, 1986]. The influence of humidity on the speed of sound was neglected at this site since the maximum measured relative humidity (RH) was 13\% (Katul, 1994). A comparison between the temperature fluctuations measured by the triaxial sonic anemometer and a fine wire Campbell Scientific type E thermocouple (TC) (0.013 mm thickness) is shown in Figure 1a. The Campbell Scientific TC does not measure the absolute air temperature. Instead, the temperature difference between the TC and an insulated junction with a thermal time constant of 20 minutes is measured [e.g., Tanner, 1988]. The TC was at the same height as the sonic anemometer but $60 \mathrm{~cm}$ to the east. Due to the limited response of the TC, the sampling frequency was set to 10 $\mathrm{Hz}$ for this comparison. The main temperature structures were captured by both instruments. We also compared the power spectra of the two temperature time series in Figure 1b. From Figure $1 b$, it is evident that both sensors agree

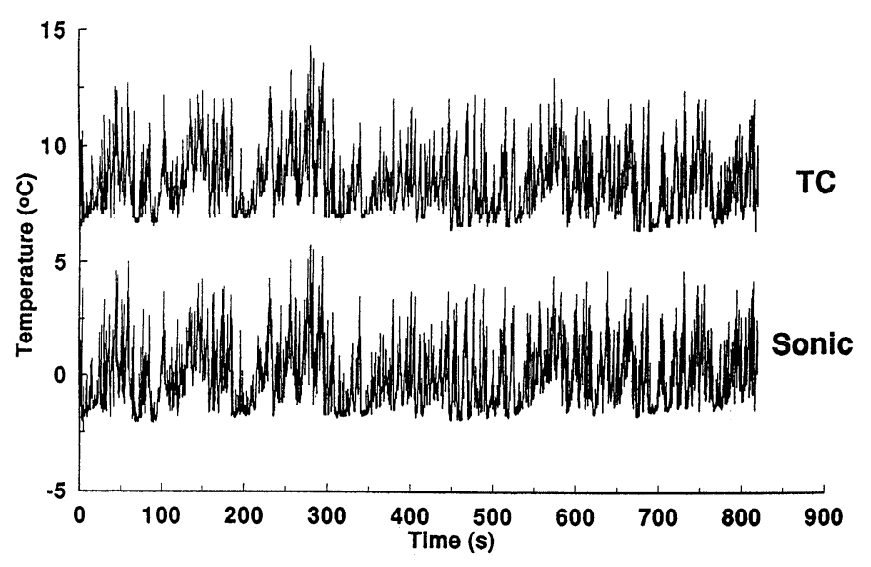

Figure 1a. Comparison between the triaxial sonic anemometer and the fine wire thermocouple (TC) temperature fluctuations measurements. The TC was placed at the same height $(=2.5$ $\mathrm{m})$ but $60 \mathrm{~cm}$ away from the sonic. For comparison purposes, the two time series are shifted by $7.5^{\circ} \mathrm{C}$. 


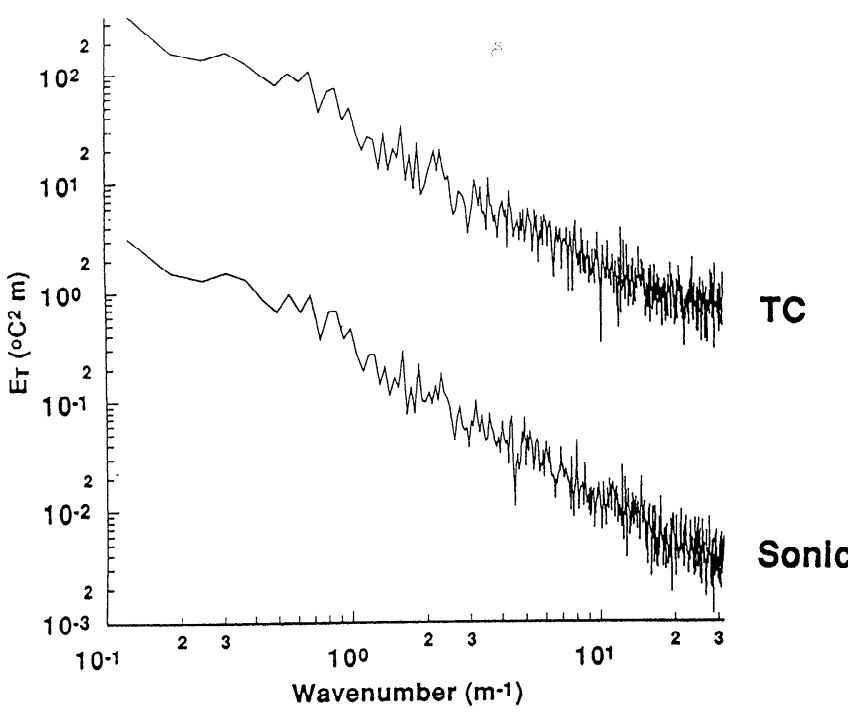

Figure 1b. Comparison between the triaxial sonic anemometer and the fine wire thermocouple (TC) temperature fluctuations power spectra. For comparison purposes, the two spectra are shifted by one decade.

very well at the low-frequency end of the spectrum; however, at the high-frequency end, the TC seems to "level off" due to limited resolution. This comparison indicates that the sonic anemometer is well suited for measuring temperature fluctuations.

\subsection{Campbell Tract}

The Campbell Tract facility is located at the University of California in Davis (elevation of $16 \mathrm{~m}$ ). The site is a uniform bare soil (Yolo clay loam) field $(500 \mathrm{~m} \times 500 \mathrm{~m})$. Experiments were conducted during August 21-23, 1993. The sonic anemometer was set at a constant height of 1.96 $\mathrm{m}$ above the ground surface. The sampling frequency was $21 \mathrm{~Hz}$, and the sampling period was 26 min resulting in 32,768 data points per velocity component. Further details regarding the experimental setup for the Campbell Tract facility are given by Katul and Parlange [1992, 1994].

\section{Results and Discussion}

One simple approach to characterize the extreme flux events or bursts in $w^{\prime} T^{\prime}$ at discrete time steps $\left(t_{i}\right)$ is through the use of an indicator function $\mathrm{I}\left(t_{i}\right)$ given by

$$
\begin{aligned}
& I\left(t_{i}\right)=1 \quad\left|w^{\prime} T^{\prime}\left(t_{j}\right)\right|>w^{\prime} T^{\prime}{ }_{c}\left(=H_{c}\right) \\
& I\left(t_{i}\right)=0 \text { otherwise }
\end{aligned}
$$

where $w^{\prime} T^{\prime}{ }_{c}=H_{c}$ is an arbitrary threshold sensible heat flux. Here, the definition of "burst" is simply an extreme flux event and is not related to the traditional definition proposed by $L u$ and Willmarth [1973] regarding bursts $\left(u^{\prime} w^{\prime}>0\right)$ in momentum fluxes. The intermittency factor $(\gamma)$ can be calculated from $I\left(t_{i}\right)$ using

$$
\gamma=\frac{1}{N} \sum_{i=1}^{i=N} I\left(t_{i}\right)
$$

where $N$ is the number of $w^{\prime} T^{\prime}$ measurements in a sampling period $(=50,400$ at Owens Lake, and 32,768 at the Campbell Tract site). Therefore $\gamma$ is the fraction of total time that the absolute sensible heat flux exceeds $w^{\prime} T_{c}^{\prime}$. The frequency of events $\left(f_{b}\right)$ exceeding $w^{\prime} T^{\prime}{ }_{c}$ is given by

$$
f_{b}=\frac{1}{N d t} \sum_{i=2}^{\mathrm{V}=N}\left(1-I\left(t_{i-1}\right)\right) I\left(t_{i}\right)
$$

where $d t$ is the sampling interval $(=1 / 56 \mathrm{~s}$ at Owens Lake, and 1/21 $\mathrm{s}$ at the Campbell Tract site). By combining (3) and (4), a mean burst persistence time $\left(\tau_{b}\right)$ is given by

$$
\tau_{b}=\frac{\gamma}{f_{b}}
$$

Analogous to many conditional sampling schemes, the choice of $H_{c}$ is rather arbitrary [Paw $U$ et al., 1992]. In this study, we depart from the classical "quadrant analysis" and focus on bursts that significantly affect the tails of the probability density function (pdf) of $w^{\prime} T$; ; hence, we selected $H_{c}$ based on the behavior of the pdf tails. This selection is done using the procedure outlined below.

1 . Normalize the $w^{\prime} T^{\prime}$ measurements by removing the mean turbulent flux $\left(\left\langle w^{\prime} T^{\prime}\right\rangle\right)$ and dividing by the standard deviation of the flux measurements $\left(\sigma_{w T}\right)$. The normalized $w$ ' $T$ ' series has a zero mean and unit variance.

2. Compute the pdf of the normalized series and compare it to a zero mean, unit variance Gaussian pdf.

3. Determine the furthest point at which the normalized $w^{\prime} T$ ' pdf tails intersects the Gaussian pdf and define this as $H_{n}$. Hence, $H_{c}=\sigma_{w T}\left(H_{n}\right)+\left\langle w^{\prime} T^{\prime}\right\rangle$.

An example is shown in Figure 2a. We performed similar analyses on all of the $w^{\prime} T^{\prime}$ signals and found that $H_{n}$ is, on the average, $2( \pm 0.34)$. Because of the relatively constant nature of $H_{n}$, we set $H_{c}=\left|\left\langle w^{\prime} T^{\prime}\right\rangle \pm 2 \sigma_{w T}\right|$ for all experiments. Notice how this criterion differs from the commonly used "hyperbolic hole" identification scheme [ e.g., Shaw, 1985; Duncan and Shuepp, 1992]. Here, the conditioning criterion depends on the variability of the flux, namely, $\sigma_{w T}$. An example of utilizing $H_{c}$ in conjunction with $I(t)$ for an individual time series, to identify extreme flux

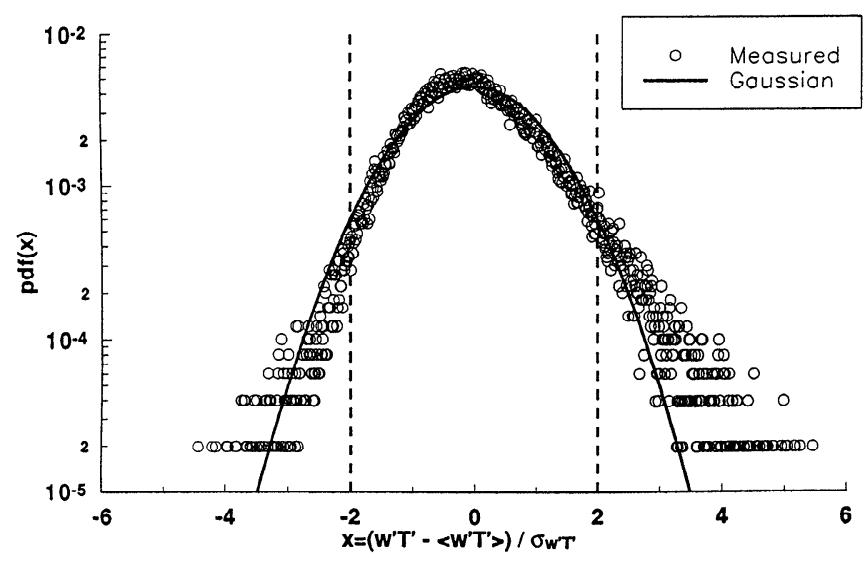

Figure 2a. Example of determining $w^{\prime} T_{c}^{\prime}\left(=H_{c}\right)$ for the hyperbolic hole quadrant analysis. The $\operatorname{pdf}(x)$ as a function of the normalized flux $(x)$ is shown. Notice how the tails significantly deviate from Gaussian at $x=2$. 


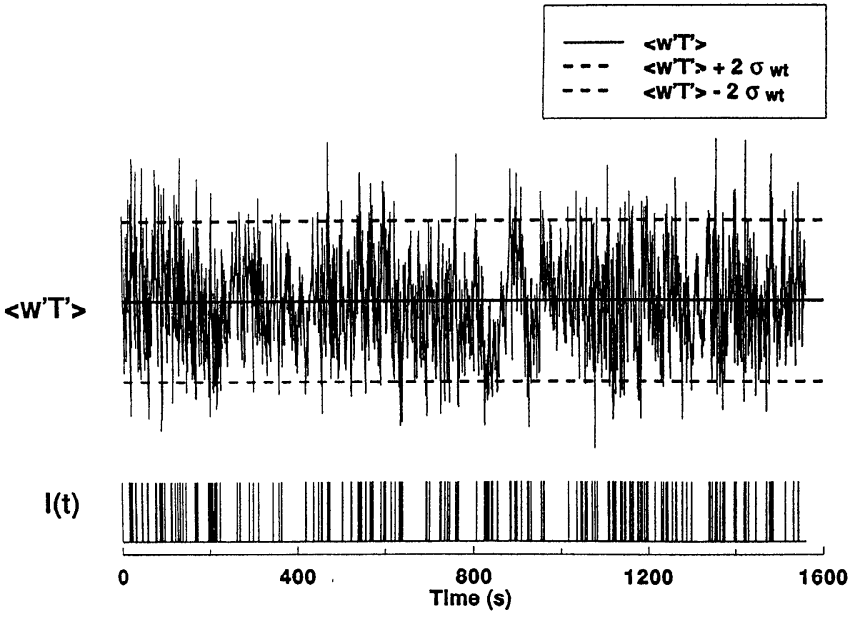

Figure 2b. The application of the hyperbolic hole for identifying extreme events in $w^{\prime} T$ ' time series. The identification is carried out by the indicator function $\left(I_{t}\right)$. For illustration purposes, the ordinate axis has arbitrary units and different scales for $w^{\prime} T$ ' and $I_{t}$.

events, is presented in Figure $2 b$. Notice in Figure $2 b$ that only a small fraction of the events are labeled as "bursts" by the $I(t)$ function.

In order to compare our $H_{c}$ with the results of other investigators, we carried out an analysis to establish an average relation between $\sigma_{w T}$ and $\left\langle w^{\prime} T^{\prime}\right\rangle$. It was found, after combining all our measurements at both sites for unstable and stable atmospheric conditions, that $\sigma_{w T}$ is about $1.9\left\langle w^{\prime} T^{\prime}\right\rangle$. Therefore $H_{c}$ is, on average, $\left.3.8<w^{\prime} T^{\prime}\right\rangle$ which agrees with the threshold $H_{c}=4\left\langle w^{\prime} T^{\prime}\right\rangle$ proposed by Shaw [1985].

\subsection{Testing the Conditioning Criterion}

The conditional sampling criterion was tested by examining the linearity between the conditioned $\left(F F_{c}\right)$ and unconditioned $(F F)$ flatness factors. The unconditioned flatness factor was computed from $F F=\left\langle\left(w^{\prime} T^{\prime}\right)^{4}\right\rangle /\left\langle\left(w^{\prime} T^{\prime}\right)^{2}\right\rangle^{2}$ while the conditioned flatness factor was computed from $\left.\mathrm{FF}_{\mathrm{c}}=\left\langle I(t)\left(w^{\prime} T^{\prime}\right)^{4}\right\rangle /<I(t)\left(w^{\prime} T^{\prime}\right)^{2}\right\rangle^{2}$ with averaging performed over nonzero values. The flatness factor was chosen due to its sensitivity to the tails of the pdf, which in our case are used to classify the extreme events. Figure 3 shows the linearity relation between the conditioned and unconditioned flatness factors $\left(R^{2}=0.96\right)$. This strong correlation indicates that $96 \%$ of the variability in $F F_{c}$ can be explained by the variability in $F F$ (and therefore, the same extreme flux events affect both $F F$ and $F F_{c}$ ). Therefore, the conditioning criterion appears to capture the contribution of extreme flux events to the tails of the flux pdf. The intermittency parameter for the heat flux measurements appears to be constant $(\gamma=0.05)$ with little variation $( \pm 0.003)$. This intermittency parameter value is much smaller than the typical value $(=0.45)$ reported by others [e.g., Priestley, 1957; Frisch and Businger, 1973; Khalsa, 1980). No significant dependence on the stability parameter $\left(z / L_{M O}\right)$ was observed. Here, $z$ is the height above the ground surface and $L_{M O}$ is the Obukhov length [e.g., Brutsaert, 1982; p. 65]. Combining both sites, the values of $\left(z / L_{M O}\right)$ varied as follows: 1) stable $\left(z / L_{M O}\right.$ between +0.12 and +1.7$\left.), 2\right)$ unstable $\left(z / L_{M O}\right.$ between -0.3 and -9.2$)$, and 3$)$ neutral $\left(\left|z / L_{M O}\right|<0.1\right)$. The lower intermittency parameter is due to the fact that our conditional sampling resolves extreme flux events that only contribute to the tails of the pdf (and therefore are rare events as shown in Figure $2 b$ ).

\subsection{PDF Properties of Bursting Duration}

We define the bursting time period $\left(L_{b}\right)$ as the duration of a single bursting event. The $L_{b}$ were determined from the time traces of $w$ 'T' collected at both sites using the following procedure.

1. Identify, from the time series of $w^{\prime} T$ ', the events where $I(t)>0$ for at least two consecutive time increments. This criterion defines a heat flux burst (see Figure 2b). A minimum of two consecutive $I(t)>0$ events were chosen simply to eliminate single bursts in the time series and to insure that some eddy motion is responsible for that event.

2. Determine the number of consecutive time increments (dt) for which the value of $I(t)>0$.

3. $L_{b}$, for this individual burst, is determined by the product of $d t$ and the number of consecutive time increments computed in step 2 .

4. Repeat steps 1-3 for all individual time traces of $w^{\prime} T$ '.

5 . The result is a vector of all possible bursting durations that defines an ensemble of $L_{b}$ values for a given site. For the combination of both sites, the ensemble exceeded 35,000 bursting events.

Using the ensemble generated from step 5, we consider the pdf of $L_{b}$ at both sites. Figure 4 shows the $\operatorname{pdf}\left(L_{b}\right)$ over four decades. It is apparent from Figure 4 that the pdf variation can be described by two power laws of the form $\operatorname{pdf}\left(L_{b}\right)=\alpha L_{b}^{-\beta}$. The possible existence of two power laws may be indicative of the bursting mechanisms. One mechanism represents the short duration but frequent bursts, while the other mechanism represents the longer duration but less

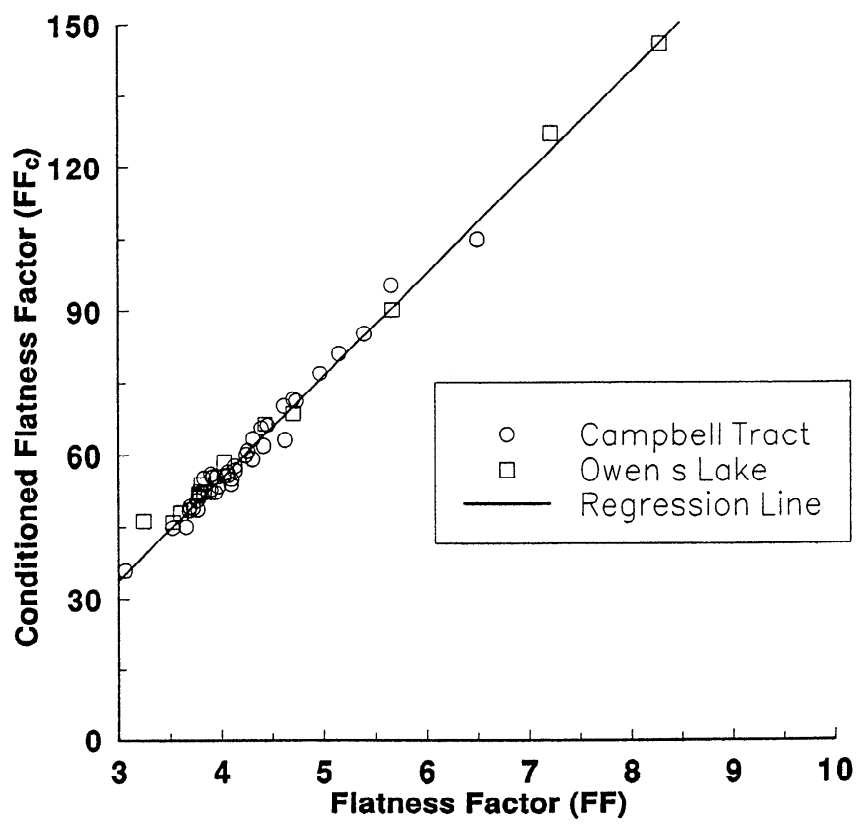

Figure 3. Comparison between conditioned $\left(F F_{c}\right)$ and unconditioned $(F F)$ flatness factors. Both $F F$ and $F F_{c}$ are strongly influenced by extreme events. 


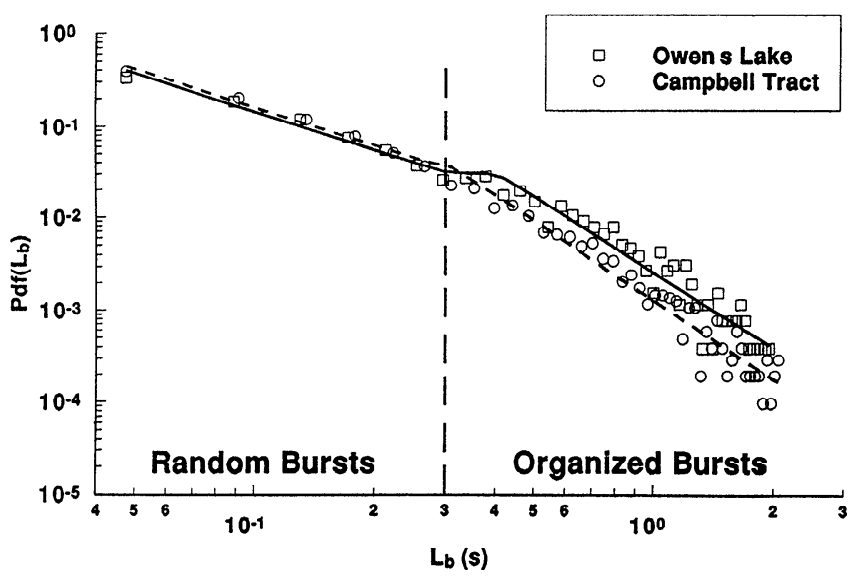

Figure 4. Variation of the probability density $\left(\operatorname{pdf}\left(L_{b}\right)\right)$ as a function of bursting duration $\left(L_{b}\right)$. The $\operatorname{pdf}\left(L_{b}\right)$ is approximated by two power laws having the same exponents at both sites. The short-dashed and solid lines are the regression fit to the $\operatorname{pdf}(x)$.

frequent bursts. The first mechanism may be due to highfrequency, less organized eddy structure in the flux measurements, while the second mechanism appears to be due to more coherent and organized large eddy motion.

Linear regression analysis was used to determine the $\alpha$ and $\beta$ of the $\operatorname{pdf}\left(L_{b}\right)=\alpha L_{b}{ }^{-\beta}$ for each site. A summary of the regression statistics is presented in Table 1 . For both bursting mechanisms $\left(L_{b}<0.3 \mathrm{~s}\right.$, and $\left.L_{b}>0.3 \mathrm{~s}\right)$ the $\beta$ 's are nearly invariant between the sites (see Figure 4 ). This near equality in $\beta$ indicates that the mechanisms responsible for bursts in the $w^{\prime} T$ ' measurements are similar at both sites and appear to be independent of stability and roughness length. It should be noted that roughness and stability conditions varied from site to site. The Campbell Tract site roughness length $\left(z_{o}\right)$ is an order of magnitude larger than that at the dry Owens lakebed and is characterized by smaller sensible heat flux. The transition $L_{b}$ from random to organized bursts is nearly the same $(\sim 0.3 \mathrm{~s})$ at each site.

Table 1. The Power-Law Behavior of the Probability Density Function Bursting Length $L_{b}$ at Each Site

\begin{tabular}{cccccc}
\hline Site & $\begin{array}{c}\mathrm{L}_{\mathrm{b}} \\
(\mathrm{s})\end{array}$ & $\log [\alpha]$ & $\beta$ & $\mathrm{R}^{2}$ & $\mathrm{SEE}$ \\
\hline $\begin{array}{c}\text { Yee et al. } \\
{[1993]}\end{array}$ & $<0.8$ & --- & $-7 / 5$ & --- & --- \\
Owens & $>0.8$ & --- & $-12 / 5$ & --- & -- \\
lake & $>0.3$ & -2.2 & -1.39 & 0.99 & 0.05 \\
$\begin{array}{c}\text { Campbell } \\
\text { tract }\end{array}$ & $<0.3$ & -2.9 & -2.74 & 0.95 & 0.11 \\
\hline
\end{tabular}

The regression model has the form $\log \left[\operatorname{pdf}\left(L_{b}\right)\right]=\log [\alpha]$ $+\beta \log \left[L_{b}\right]$. The transition $L_{b}$ at which the power-law exponent changes is $0.3 \mathrm{~s}$. The coefficients of determination $\left(R^{2}\right)$ and the standard errors of estimate (SEE) are also shown. For comparison purposes, the values reported by Yee et al. [1993] are tabulated.
The values of $\beta$ obtained in Table 1 for both regimes are in agreement with the values reported by Yee et al. [1993] for concentration fluctuation measurements in a dispersing plume from an elevated point source. The data set collected by Yee et al. [1993] was obtained from 30 field experiments with sampling durations ranging from 16 to $64 \mathrm{~min}$ and sampling frequency of $1 \mathrm{KHz}$. Although it appears that their experiments werc carricd out over a moderate range of stability regimes (nighttime and daytime), no explicit values were given for $z / L_{M O}$.

Yee et al. [1993], using a different conditional sampling scheme, also found that the pdf of the concentration burst length exhibits two distinct power law regimes. In the first regime their $\operatorname{pdf}\left(L_{b}\right)$ is proportional to $L_{b}{ }^{-7 / 5}$, and in the second regime their $\operatorname{pdf}\left(L_{b}\right)$ is proportional to $L_{b}^{-12 / 5}$. Compare these power laws with the $\beta$ reported in Table 1. Another interesting point to compare with Yee et al. [1993] is the cutoff between the two regimes. They reported a cutoff, marking the transition from one regime to the next, of about $1 \mathrm{~s}$, while the cutoff is $0.3 \mathrm{~s}$ for this study. They suggested that the second regime is due to plume meandering, (which is a coherent large scale entity), while the first regime is associated with the nibbling of small-scale motion (inplume fine structure) superimposed on the large-scale eddy motions. These regimes are analagous to the two bursting mechanisms described earlier for the sensible heat flux. Another conclusion of Yee et al. [1993] is the invariance of the exponents of the two bursting regimes across the mean plume cross section. Their results may indicate some homogeneity in the exponent statistics.

\subsection{The Influence of Bursting on Flux Integral Timescales}

It is desirable to relate the frequency of flux bursts to a characteristic timescale representing the influence of large eddy motion. For that purpose, we compared the integral

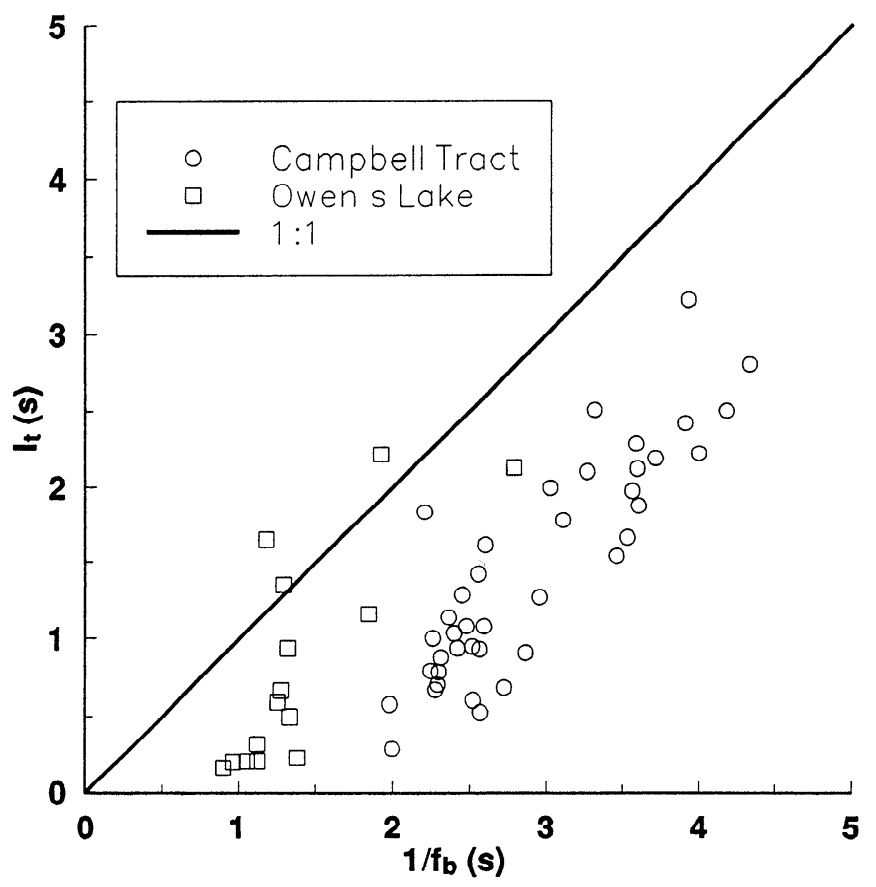

Figure 5. Relation between bursting frequency $\left(f_{b}\right)$ and the flux integral time scale $\left(I_{t}\right)$. The 1:1 line is also shown. 
Table 2. Relation Between Bursting Separation Time $\left(1 / f_{b}\right)$ and the Integral Time Scale $\left(I_{t}\right)$

\begin{tabular}{lll}
\hline $\begin{array}{l}\text { Regression } \\
\text { Statistics }\end{array}$ & $\begin{array}{l}\text { Owens } \\
\text { Lake }\end{array}$ & Campbell Tract \\
\hline Slope (A) & +1.00 & +0.95 \\
Intercept (B) & -0.57 & -1.19 \\
$\mathrm{R}^{2}$ & +0.76 & +0.75 \\
SEE (s) & +0.46 & +0.34 \\
\hline
\end{tabular}

A linear regression model of the form $I_{t}=A\left(1 / f_{b}\right)+B$ is used to assess the relationship. The coefficients of determination $\left(\mathbf{R}^{2}\right)$ and the standard errors of estimate (SEE) are also shown.

timescale $\left(I_{t}\right)$ of the flux measurements to a characteristic burst timescale $\left(f_{b}^{-1}\right)$ in Figure 5. It should be noted that $\mathrm{I}_{\mathrm{t}}$ is a timescale that is independent of location or duration of burst events (i.e., $I_{t}$ is a nonlocal statistic), while $1 / f_{b}$ is a mean time interval between bursts (i.e. $1 / f_{b}$ is a local statistic).

The integral timescale is computed from the area under the flux autocorrelation function up to the first zero crossing (see Sirivat and Warhaft [1983] for further details regarding advantages of truncating the autocorrelation function at the first zero crossing). Figure 5 indicates a strong relation $\left(R^{2}=0.75\right)$ between the integral timescale and the frequency of bursts (see also Table 2). A regression model of the form $I_{t}=\mathrm{A}\left(1 / f_{b}\right)+B$ was used to examine possible relation between $I_{t}$ and $\left(1 / f_{b}\right)$. Notice from Table 2 that the slope A is nearly unity for both Owens lake and the Campbell Tract data. This relation between $I_{t}$ and $\left(1 / f_{b}\right)$ indicates that the main contribution to sensible heat flux internal correlation is bursting. The fact that $I_{t}$ is finite and is related to the bursts is critical for choosing proper flux sampling periods $\left(\tau_{s}\right)$. As shown by Lumley and Panofsky [1964l and Lumley [1970] the relation between sampling intervals, errors in approximating ensemble averages by time averages $(\epsilon)$, and $I_{t}$ is where $w$ and $T$ are the instantaneous vertical velocity and temperature, respectively. Because bursting increases both

$$
\tau_{s}=\frac{\left.2<\left(w^{\prime} T\right)^{2}\right\rangle}{\langle(w T)\rangle^{2}} \frac{I_{t}}{\epsilon^{2}}
$$

$\left\langle\left(w^{\prime} T^{\prime}\right)^{2}\right\rangle$ and $I_{t}$, the flux sampling period necessary for the convergence of ensemble averages to time averages becomes large.

\subsection{The Influence of Stability on Bursting Frequency}

The strong correlation between $I_{t}$ and $\left(1 / f_{b}\right)$ suggest significant interaction between the turbulent production mechanism (through $I_{t}$ ) and the bursting frequency. In the ASL, we note that both shear and buoyancy are responsible for generating turbulence, and thus a variety of timescales can be used to normalize $\left(1 / f_{b}\right)$. For that purpose, we define three timescales

$$
\begin{array}{lll}
t_{s h}=z & u_{*}^{-1} \\
t_{b u}=z & w_{*}^{-1} \\
t_{R}=z & R_{*}^{-1}
\end{array}
$$

corresponding to three velocity scales

$$
\begin{aligned}
& u_{*}=<-u^{\prime} w^{\prime}>^{1 / 2} \\
& w_{*}=\left(\frac{g}{c_{p} T_{a}}\left|<w^{\prime} T^{\prime}>\right| z\right)^{1 / 3} \\
& R_{*}=\left(u_{*}^{2}+w_{*}^{2}\right)^{1 / 2}
\end{aligned}
$$

where $u_{*}$ is the friction velocity, $u^{\prime}$ is the longitudinal velocity fluctuation, and $z$ is the height from the ground surface. The velocities in (8) represent characteristic velocity scales for shear (longitudinal), buoyancy (vertical), and composite shear buoyancy (two dimensional). Figure 6 shows the mean bursting frequency at both sites, normalized by the three velocity scales, as a function of the stability parameter $\left(z / L_{M O}\right)$ for a wide range of unstable and stable atmospheric conditions. The following can be noted from Figure 6.

1. Irrelevent of the normalization scheme, the mean interval time between bursts is longer for unstable stability when compared to stable stability.

2 . The influence of stability variation on the mean interval time between bursts is very significant for $0<-z / L_{M O}<3$.

3 . The influence of stability variation on the mean interval between bursts is small for near-convective atmospheric stability conditions.

These points suggest that atmospheric stability variation influences the mean interval between bursts for the dynamic and dynamic convective sublayers of the ASL. These sublayers are characterized by strong to moderate shear production of turbulence (see Kader and Yaglom [1990]) for description of statistical properties of the dynamic and dynamic convective sublayers). Similar findings regarding the role of shear were noted by Antonia et al. [1979] for temperature.
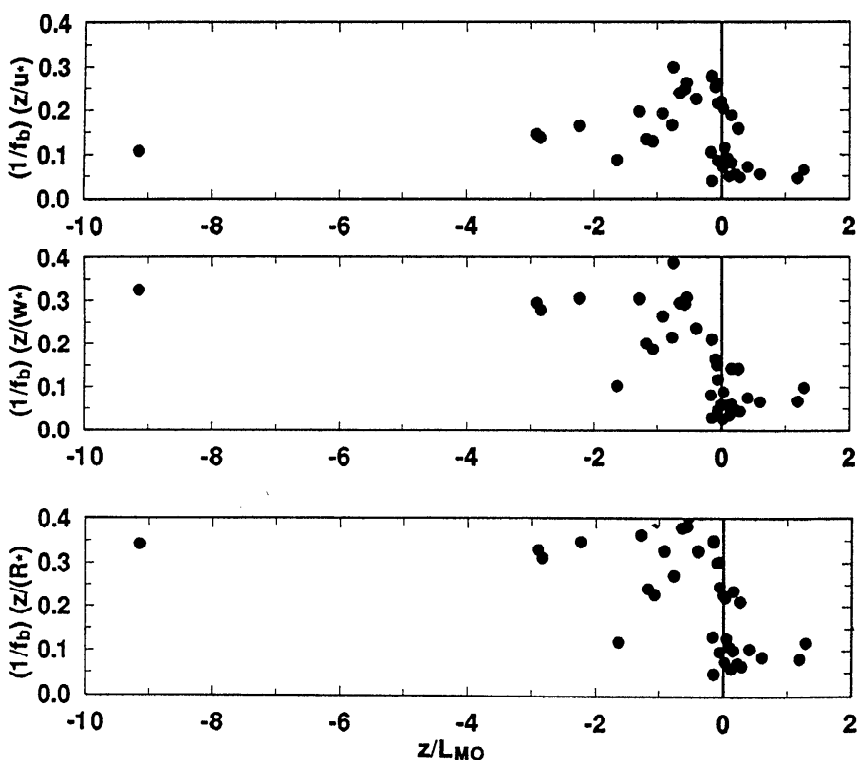

Figure 6. The mean time interval between bursts, normalized by a turbulent production timescale, as a function of atmospheric stability conditions. The turbulent production timescales are shear (upper figure), buoyancy (intermediate figure), and combination (lower figure). 


\section{Conclusions}

This study has examined the temporal structure of sensible heat flux using a modified hyperbolic hole quadrant analysis. The sensible heat flux time series measurements were collected over two sites having different roughness lengths and sensible heat flux conditions. This analysis has focused on the duration and frequency of the extreme sensible heat flux bursts, as identified by a modified hyperbolic hole method. The identification scheme was tested by comparing the conditioned and unconditioned flatness factors to extreme events. Subsequently, all the extreme events were combined into two large ensembles that represent all measured bursting durations at each site. The probability density functions of the bursting duration exhibit two distinct power laws. The exponents of the power laws were computed and compared with turbulence concentration measurements in dispersing plumes. The agreement between the exponents computed from dispersing plume experiments and our experiments was within $10 \%$. More experiments over a variety of different surfaces and stability conditions are necessary to investigate possible universal behavior of these power law exponents. It was also found that the flux integral timescale is strongly correlated with the bursting frequency $\left(R^{2}=0.75\right)$. This correlation suggests interaction between the mean time interval between bursts and the turbulence production mechanism. It was found that the mean interval between bursts is smaller for stable conditions when compared to unstable conditions. Within the dynamic and dynamic convective sublayers of the atmospheric surface layer, the variation of the mean time interval between bursts is strongly dependent on the stability parameter $\left(z / L_{M O}\right)$. This was not observed for the free convective sublayer.

Acknowledgments. The authors would like to thank Mike Mata for his help in maintaining the field site, Han Stricker and Teresa Ortenburger for their assistance during the experiment, and Scott Tyler for his support at Owens lake. We are grateful for the funding support from the National Science Foundation (NSF) under grant EAR-93-04331, U.S. Geological Survey (USGS), Water Resources Center (WRC) under grant W-812, Kearney Foundation, and UC Davis superfund grant 5 P42ESO4699-07.

\section{References}

Antonia, R.A., A.J. Chambers, C.A. Friehe, and C.W. Van Atta, Temperature ramps in the atmospheric surface layer, J. Atmos. Sci., 36, 99-108, 1979.

Antonia, R.A., Conditional sampling in turbulence measurement, Annu. Rev. Fluid Mech., 13, 131-156, 1981.

Blackwelder, R.F., and R.E. Kaplan, 1976, On the wall structure of turbulent boundary layer, J. Fluid Mech., 76, 89-112, 1976.

Brutsaert, W., Evaporation Into the Atmosphere: Theory, History, and Applications, 299 pp., Kluwer Academic, Hingham, Mass., 1982.

Chen, C.C., and R.F. Blackwelder, Large-scale motion in a turbulent boundary layer: A study using temperature contamination, J. Fluid Mech., 89, 1-31, 1978.

Dickinson, R.E., Land-surface processes and climatesurface albedos and energy balance, $A d v$. Geophy., 25, 305$353,1983$.

Duncan, M.R., and P.H. Schuepp, A method to delineate extreme structures within airborn flux traces over the FIFE site, J. Geophys. Res., 97, 18487-18498, 1992.

Friehe, C.A., Fine-scale measurements of velocity, temperature, and humidity in the atmospheric boundary layer, in Probing the Atmospheric Boundary Layer, edited by D.H. Lenschow, American Meteorological Society, Boston, Mass., pp. 29-38, 1986.

Frisch, S., and J.A. Businger, A study of convective elements in the atmospheric surface layer, Boundary Layer Meteorol., 3, 301-328, 1973.

Gao, W., R.H. Shaw, and K.T. Paw U, Conditional analysis of temperature and humidity microfronts and ejection/sweep motions within and above a deciduous forest, Boundary Layer Meteorol., 59, 35-57, 1992.

Grossman, R.L., Bivariate conditional sampling of moisture flux over a tropical ocean, J. Atmos. Sci., 41, 3238-3253, 1984.

Kader, B.A., and A.M. Yaglom, Mean fields and fluctuation moments in unstably stratified turbulent boundary layers, J. Fluid Mech., 212, 637-662, 1990.

Katul, G.G., A model for sensible heat flux probability density function for near-neutral and slightly-stable atmospheric flows, Boundary Layer Meteorol., in press, 1994.

Katul, G.G., and M.B. Parlange, A Penman-Brutsaert model for wet surface evaporation, Water Resour. Res. , 28, 121-126, 1992.

Katul, G.G., and M.B. Parlange, On the active role of temperature in surface layer turbulence, J. Atmos. Sci., 51, 2181-2195, 1994.

Katul, G.G., M.B. Parlange, and C.R. Chu, Intermittency, local isotropy, and non-Gaussian statistics in atmospheric surface layer turbulence, Phys. Fluids, 6, 2480-2492, 1994.

Khalsa, S.J.S., Surface-layer intermittency investigated with conditional sampling, Boundary Layer Meteorol., 19, 135-153, 1980.

Lu, S.S., and W.W. Willmarth, Measurements of the structure of the Reynolds stress in a turbulent boundary layer, J. Fluid Mech., 60, 481-512, 1973.

Lumley, J.L., Stochastic Tools in Turbulence, 194 pp., Academic Press, San Diego, Calif., 1970.

Lumley, J., and H. Panofsky, The Structure of Atmospheric Turbulence, 239 pp., John Wiley, New York, 1964.

Mahrt, L., Eddy asymmetry in the shear heated boundary layer, J. Atmos. Sci., 48, 472-492, 1991.

Nakagawa, H., and I. Nezu, Prediction of the contributions to the Reynolds stress from bursting events in open channel flows, J. Fluid Mech., 80, 99-128, 1977.

Nakagawa, H., and I. Nezu, Structure of space-time correlations of bursting phenomena in an open-channel flow, J. Fluid Mech., 104, 1-43, 1981.

Paw U, K.T., Y. Brunet, S. Collineau, R.H. Shaw, T. Maitani, J. Qiu, and L. Hipps, On coherent structures in turbulence above and within agricultural canopies, Agric. For. Meteorol., 61, 55-68, 1992.

Priestley,.H.B., Convection from the Earth's surface, Proc. Roy. Soc. London A, 238, 287-304, 1957.

Raupach, M.R., Conditional statistics of Reynolds stress in rough and smooth-wall turbulent boundary layers, J. Fluid Mech., 108, 363-382, 1981.

Raupach, M.R., R.A. Antonia, and S. Rajagopalan, rough-wall turbulent boundary layers, Appl. Mech. Rev., 44, 1-25, 1991.

Sellers, P.J., Biophysical models of land surface processes, in Climate System Modeling, edited by Trenberth, K.E., pp. 451-490, Cambridge University Press, New York, 1992.

Shaw, R.H., On diffusive and dispersive fluxes in forest canopies, in The Forest-Atmosphere Interaction, edited by B.A. Hutchison, and B.B. Hicks, D. Reidel Publishing Co., Dordrecht, The Netherlands, 407-419, 1985. 
Shaw, R.H., J. Tavangar, and D.P. Ward, Structure of the Reynold stress in a canopy layer, J. Clim. Appl. Meteorol., 22, 1922-1931, 1983.

Sirivat, A., and Z. Warhaft, The effect of a passive cross-stream temperature gradient on the evolution of temperature variance and heat flux in grid generated turbulence, J. Fluid Mech., 128, 323-346, 1983.

Suomi, V.E., and J.A. Businger, Principle of the sonic anemometer-thermometer, Geophys. Res. Pap., 59, 1-13, 1959.

Tanner, B.D., Use and requirements for Bowen ratio and eddy correlation determination of evapotranspiration, paper presented at Specialty Conference of Irrigation and Drainage Division, Am. Soc. of Civ. Eng., Lincoln, Neb., July 19-21, 1988.

Wyngaard, J.C., Cup, propeller, vane, and sonic anemometers in turbulence research, Annu. Rev. Fluid Mech., 13, 399-423, 1981.
Yee, E., P.R. Kosteniuk, G.M. Chandler, C.A. Biltoft, and J.F. Bowers, Statistical characteristics of concentration fluctuations in dispersing plumes in atmospheric surface layer, Boundary Layer Meteor., 65, 69-109, 1993.

J. Albertson and M. Parlange, Hydrologic Science, University of California, Davis, CA 95616.

C.R. Chu, Department of Civil Engineering, National Central University, Chung-Li, Taiwan.

G. Katul, School of the Environment, Duke University, Durham NC 27708-0328.

H. Stricker, Department of Water Resources, Agricultural Engineering, Wageningen, Netherlands.

(Received November 9, 1993; revised June 21, 1994; accepted June 23, 1994.) 